\title{
Synergistic effect of a tissue kallikrein 1 and tissue inhibitor of matrix metalloproteinase 1 co-expression vector on the proliferation of rat vascular smooth muscle cells
}

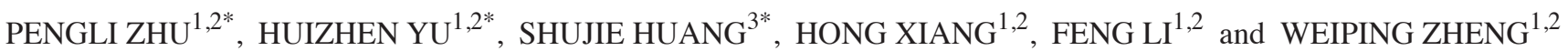 \\ ${ }^{1}$ Department of Geriatrics, Fujian Provincial Hospital Key Laboratory of Geriatrics, Fujian Medical University, \\ Fuzhou, Fujian 350001; ${ }^{2}$ Fujian Institute of Clinical Geriatrics, Fuzhou, Fujian 350001; ${ }^{3}$ Department of Cardiology, \\ Fujian Provincial Hospital, Fuzhou, Fujian 350001, P.R. China
}

Received September 13, 2014; Accepted June 22, 2015

DOI: $10.3892 / \mathrm{mmr} .2015 .4198$

\begin{abstract}
Tissue kallikrein 1 (TK1) and tissue inhibitor of matrix metalloproteinase 1 (TIMP1) are important in inhibiting vascular smooth muscle cell (VSMC) proliferation and improving vascular remodeling, respectively. It was hypothesized that a combination of TK1 and TIMP1 genes, mediated by an adenovirus vector could augment or act in synergy to enhance the inhibitory effects. The promoter, mCMV carrying hTIMP1 cDNA was subcloned into pDC316-hTK1 to construct a recombinant plasmid carrying hTK1 and hTIMP1 genes. Subsequently, the double gene plasmid and adenovirus backbone plasmid were packaged into HEK293A cells. Gene transcription and protein expression were examined, respectively using reverse transcription-quantitative polymerase chain reaction (PCR) and western blotting assays. VSMC proliferation was assessed using cell counting and methyl-thiazolyl-tetrazoliuin methods. The constructed plasmid containing hTK1 and hTIMP1 genes was correctly identified by means of PCR, double digestion and sequencing analysis. The co-expression vector, Ad-hTK1-hTIMP1 was successfully constructed and packaged into HEK293A cells. When VSMCs were transfected with the co-expression vector, the mRNA transcription and protein expression of hTK1 and hTIMP1 exhibited abundant expression in a concentration-dependent and time-dependent manner, independently. In conclusion, the co-expression vector synergistically inhibited
\end{abstract}

Correspondence to: Professor Pengli Zhu, Department of Geriatrics, Fujian Provincial Hospital Key Laboratory of Geriatrics, Fujian Medical University, 134 East Street, Fuzhou, Fujian 350001, P.R. China

E-mail: zpl7755@126.com

*Contributed equally

Key words: human tissue kallikrein 1, human tissue inhibitor of metalloproteinase 1, gene therapy, vascular smooth muscle cell, cell proliferation the cell growth and proliferation induced by platelet-derived growth factor-BB compared with the single gene vector.

\section{Introduction}

Tissue kallikrein 1 (TK1) cleaves low-molecular-weight kininogen to release bradykinin and Lys-bradykinin (kallidin), which exert biological functions via kinin receptor signaling (1,2). All components of the tissue kallikrein-kinin system have been identified in the cardiovascular system. Using various delivery approaches of TK1 protein, numerous studies have demonstrated that TK1 exhibits a wide spectrum of beneficial effects through kinin B2 receptor signaling by reducing cardiac remodeling, renal injury and ischemic stroke, and preventing long-term in-stent restenosis (3-6). Our previous studies in vitro and in vivo, revealed that the effects of human TK1 (hTK1) gene delivery inhibited vascular smooth muscle cell (VSMC) proliferation and partially inhibited neointima formation following carotid artery injury in rats $(7,8)$.

The extracellular matrix (ECM) is responsible for the three-dimension spatial arrangement and structural integrity of the arterial wall, and the metabolic function of intracellular components. Alterations have been reported in the density, architecture and composition of the ECM in vessels as a result of hypertension $(9,10)$. Matrix metalloproteinases (MMPs) and tissue inhibitors of metalloproteinases (TIMPs) are vital in the regulation of ECM metabolism in normal and pathological conditions (11). MMP-9 digests gelatin, elastin, fibronectin, laminin, and types IV and V collagen, which are found in the subendothelial basement membranes $(11,12)$. TIMP-1 blocks the activation of MMPs, preventing their proteolytic activity. MMPs and TIMPs regulate the metabolism of collagen and elastin and are, therefore, responsible for structural and functional alterations in the arterial wall during vascular remodeling $(11,12)$. TIMP-1 has been demonstrated to inhibit the process of vascular remodeling in vitro, and overexpression of this gene demonstrates potential for destabilizing vessel differentiation (13). Delivery of the adenovirus vector cDNA encoding TIMP-1 could partly restrain VSMC proliferation and migration, and therefore reduce neointimal hyperplasia in a rat model of vascular balloon injury (14). 
At present, there are two main methods for multigene therapy. The first method involves the target cells being transfected with multiple independent vectors carrying different genes simultaneously $(15,16)$. The second method involves the co-expression of multiple genes in one identical vector (17). Compared with the first method, the use of a multigene co-expression vector may increase the efficiency of transfection and expression. The low efficiency of gene transfer is the bottleneck in gene therapy at present. In theory, a combination of two or more anti-restenosis genes carried by a single vector could improve treatment efficacy, reduce the side effects associated with vectors, and have potential for clinical application (18). However, these methods have seldom been investigated in the context of cardiovascular disease.

In previous studies, TK1 and TIMP1 have been observed to have numerous biological effects on vascular remodeling. The synergistic suppression of a conjunction of TK1 and TIMP1 for VSMC proliferation remains to be elucidated. The aim of the present study was to construct an adenovirus vector containing human TK1 and TIMP1 genes. The vector would be used for the co-expression of TK1 and TIMP1 proteins, and provide a novel strategy for inhibiting VSMC proliferation.

\section{Materials and methods}

Plasmid and recombinant adenovirus. Plasmid pDC316, adenoviral skeleton plasmid pBHGloxE1, 3Cre and DH5- $\alpha$ were purchased from Mixcrobix-Biosystems (Toronto, ON, Canada). Plasmid pDC316-hTIMP1-enhanced green fluorescent protein (EGFP), which contains the mCMV promoter and hTIMP1 cDNA, pDC316-hTK1, which contains hTK1 cDNA, and recombinant adenovirus Ad5-hTK1-IRES-EGFP (Ad-hTK1) and Ad-hTIMP1-EGFP (Ad-hTK1), as well as control vector Ad-EGFP were constructed and maintained in our laboratory (Fujian Provincial Hospital Key Laboratory of Geriatrics, Fuzhou, China). Rabbit anti-hTK1 monoclonal antibodies and rabbit anti-hTIMP1 polyclonal antibodies were purchased from Abcam (Cambridge, MA, USA). UV transilluminator was purchased from Jingke Scientific Instrument Co., Ltd. (Shanghai, China). AdMax system was obtained from Microbix Biosystems, Inc. (Mississauga, Canada).

Construction of recombinant plasmid containing hTK1 and hTIMP1 genes. The mCMV-hTIMP1 fragment from constructed pDC316-mCMV-hTIMP1 was amplified using polymerase chain reaction (PCR) with the following primers: mCMV-BglII, forward 5'-GCCAGATCTGTTGACATTGAT TATTGA-3', hTIMP1-SalI and reverse 5'-GCCGTCGACTCA GGCTATCTGGGACCG-3'. This pair of primers contained restriction sites for $\mathrm{Bg} / \mathrm{II}$ and $\mathrm{SalI}$ at the 5 ' terminal, respectively. The PCR reaction system (Bio-Rad Laboratories, Inc., Hercules, CA, USA) contained primer 1, primer 2, dNTP and pyrobest Taq DNA polymerase (Takara Bio, Inc., Otsu, Japan). The PCR reaction program was accomplished as follows: Initial denaturation at $94^{\circ} \mathrm{C}$ for $5 \mathrm{~min}$ followed by $94^{\circ} \mathrm{C}$ for $30 \mathrm{sec}, 57^{\circ} \mathrm{C}$ for $40 \mathrm{sec}$ for annealing and $72^{\circ} \mathrm{C}$ for $80 \mathrm{sec}$ for extension, which was repeated for 30 cycles, with an extra final $5 \mathrm{~min}$ incubation at $72^{\circ} \mathrm{C}$ to complete all extensions.
The loxP-containing shuttle vector pDC316-hTK1 contains hTK1 cDNA driven by the mCMV promoter, which was verified and maintained in our lab (7). The mCMV-hTIMP1 PCR fragment was purified using a PCR purification kit (Takara Bio, Inc.) and then double-digested using $B g l \mathrm{II}$ and $\mathrm{SalI}$ restriction enzymes (Takara Bio, Inc.). Subsequently, the mCMV-hTIMP1 fragment was cloned into pDC316-hTK1, the digestion products of which were identified by gel electrophoresis, prior to being recycled and ligated by T4 DNA ligase (Takara, Bio, Inc.). The ligation products underwent a heat shock reaction in a $42^{\circ} \mathrm{C}$ water bath for $90 \mathrm{sec}$, and were quickly transferred onto ice for $2 \mathrm{~min}$. The ligation products were then transformed into competent Escherichia coli DH5- $\alpha$ (American Type Culture Collection, Manassas, VA, USA) for $24 \mathrm{~h}$. Various clones were screened using the colony PCR method. The positive clones and extracted plasmids were analyzed using PCR, digestion using $B g l$ II and SalI and sequencing (Invitrogen Life Technologies, Carlsbad, CA, USA) using an mCMV-hTIMP1-F primer.

Packaging and amplification of recombinant adenovirus. Briefly, HEK293A cells (American Type Culture Collection) at a confluence of $40-60 \%$ were co-transfected with a recombinant plasmid containing hTK1 and hTIMP1 cDNA and pBHGlox DE1, 3Cre with the aid of Lipofectamine 2000 (Invitrogen Life Technologies) according to standard methods, as described previously $(7,19)$. High titer adenovirus was obtained by repeated infection in HEK293A cells. The recombinant adenoviral vectors containing target cDNA were screened and verified using PCR with the two primers. The adenovirus was amplified and purified using standard viral amplification and cesium chloride purification methods $(7,20)$.

The viral titer, expressed as transducing units per milliliter, was determined using a dilution assay in HEK293A cells that were transduced with a serial dilution of concentrated adenovirus. The viral titer was detected by a TCID50 assay provided by Obiogene, Inc. (Carlsbad, CA, USA). Following purification, the virus was suspended in phosphate-buffered saline (PBS) with $3 \%$ sucrose and preserved at $-80^{\circ} \mathrm{C}$ until use.

VSMC culture and transfection with co-expression vector. Rat primary VSMCs were isolated from the thoracic aorta of Sprague-Dawley (SD) rats (2-3-month-old) using the explant method as described previously (21), and then cultured in Dulbecco's modified Eagle's medium (DMEM; Gibco Life Technologies, Carlsbad, CA, USA) containing 10\% fetal bovine serum (FBS; Gibco Life Technologies), $100 \mathrm{U} / \mathrm{ml}$ penicillin, and $100 \mathrm{U} / \mathrm{ml}$ streptomycin (Gibco Life Technologies). The cells were incubated at $37^{\circ} \mathrm{C}$ in a humidified atmosphere of $5 \% \mathrm{CO}_{2}$. The cells exhibited a spindle-shaped appearance, and confluent VSMCs in culture exhibited the characteristic 'hill and valley' growth pattern (7). The cell morphology was examined in detail under an inverted phase contrast microscope (DMI4000B; Leica Microsystems, Wetzlar, Germany) at 100x magnification. VSMCs were used for experiments between the $3 \mathrm{rd}$ and 7 th passages. SD rats were obtained from the Experimental Animal Center of the Fujian Medical University (Fuzhou, China). The study was approved by the Institutional Ethics Committee for Laboratory Animal Care of Fujian Provincial Hospital (Fuzhou, China). 
VSMCs were seeded in 6-well culture plates at a confluence of 30-40\%, and the adenovirus of Ad-hTK1, Ad-hTIMP1, Ad-hTIMP1-hTK1 and Ad-EGFP at different multiplicities of infection (MOI) were added to the medium. The adenovirus was incubated with cells in a small volume of serum-free medium at $37^{\circ} \mathrm{C}$. After absorption for $2 \mathrm{~h}$, fresh complete growth medium was added and the cells were further incubated for the following experiments. After $72 \mathrm{~h}$ of infection, adenovirus infectivity was measured using EGFP with a fluorescent microscope (DMI4000B; Leica Microsystems) at 100x magnification. Reverse transcription-quantitative (RT-q) PCR and western blot analyses were performed to detect the expression of hTK1 and hTIMP1 mRNA and protein.

$R T-q P C R$. Total RNA was extracted using RNAiso Plus (Takara Bio, Inc.) and stored at $-80^{\circ} \mathrm{C}$ until further use. The concentration and purity of RNA was measured under a UV spectrophotometer (ND-2000; Thermo Fisher Scientific, Inc., Wilmington, DE, USA). Reverse transcription was conducted to synthesize first-strand cDNA from $1 \mu \mathrm{g}$ total RNA using the PrimeScript RT Reagent kit with gDNA Eraser (Takara Bio, Inc.), according to the manufacturer's instructions. The resulting cDNA was then subjected to RT-qPCR, in order to evaluate the relative mRNA expression levels of hTK1 and hTIMP1. $\beta$-actin was used as an internal control. Gene-specific amplification was performed using a Lightcycler 480 Real Time PCR system (Bio-Rad Laboratories, Inc.) with $12.5 \mu 1$ SYBR PremixEx Taq II, $1.0 \mu \mathrm{l}$ PCR forward $(10 \mu \mathrm{M})$ and $1.0 \mu \mathrm{l}$ PCR reverse primer (10 $\mu \mathrm{M}$; Invitrogen Life Technologies), $2.0 \mu \mathrm{l}$ cDNA, $8.5 \mu 1$ raze-free water. The PCR reaction mix was pre-heated to $95^{\circ} \mathrm{C}$ for $30 \mathrm{sec}$, and then amplified at $95^{\circ} \mathrm{C}$ for $5 \mathrm{sec}$ and $60^{\circ} \mathrm{C}$ for $30 \mathrm{sec}$ for 40 cycles. The resolution curve was measured at $95^{\circ} \mathrm{C}$ for $15 \mathrm{sec}, 60^{\circ} \mathrm{C}$ for $15 \mathrm{sec}$ and $95^{\circ} \mathrm{C}$ for $15 \mathrm{sec}$. The $\mathrm{Ct}$ (threshold cycle) value of each sample was calculated using the instrument's software, and the relative mRNA expression levels of total hTK1 and hTIMP1 mRNA were normalized to those of $\beta$-actin. The data were analyzed using the $2^{-\Delta \Delta \mathrm{Ct}}$ method. The following primer sequences were used: hTK1, forward 5'-GTCCAGAACAATCCGCCTCA-3', reverse 5'-CAGACCACCATGCCCAGTTAGA-3'; hTIMP1, forward 5'-CCTTATACCAGCGTTATGAGATCAA-3', reve rse 5'-AGTGATGTGCAAGAGTCCATCC-3'; and $\beta$-actin, forward 5'-TGGCACCCAGCACAATGAA-3', and reverse 5'CTAAGTCATAGTCCGCCTAGAAGCA-3'.

Western blot analysis. Cells were lysed in a buffer containing Triton X-100 (Beijing Solarbio Science \& Technology Co., Ltd., Beijing, China), separated by $12 \%$ SDS-PAGE (Beyotime Institute of Biotechnology, Jiangsu, China), and blotted onto nitrocellulose membranes (Merck Millipore, Carrigtwohill, Ireland). To detect hTK1 and hTIMP1, the membranes were blocked with $5 \%$ non-fat dry milk in tris-buffered saline (TBS) containing $0.1 \%$ Tween-20 (Beyotime Institute of Biotechnology) at room temperature for $2 \mathrm{~h}$. The membranes were then incubated overnight at $4{ }^{\circ} \mathrm{C}$ with monoclonal rabbit anti-rat hTK1 (1:5,000; cat. no. ab76495; Abcam), polyclonal rabbit anti-rat hTIMP1 (1:5,000; cat. no. ab61224; Abcam) or monoclonal rabbit anti-rat $\beta$-actin (1:1,000; cat. no. 4970; Cell Signaling Biotechnology, Inc., Danvers, MA, USA). Following washing, the membranes were incubated for $2 \mathrm{~h}$ at room temperature with an anti-rabbit horseradish peroxidase-conjugated secondary antibody (1:5,000; cat. no. sc2030; Santa Cruz Biotechnology, Inc., Dallas, TX, USA). Subsequently, the membranes were washed five times in TBS with $0.1 \%$ Tween-20, and target proteins bands were detected using a horseradish-linked immunoassay with an enhanced chemiluminescence western blotting system (CWBIO, Beijing, China). The relative intensity of the bands of interest were analyzed with relative protein analytic software (Imaje J 1.32; National Institutes of Health, Bethesda, MD, USA) $(n=3)$.

Cell proliferation assay. To determine whether the adenovirus Ad-hTIMP1-hTK1 is biologically active, two different methods were used, cell counting and a 3-[4,5-dimethy1-2-thiazolyl]-2,5-dipheny1- 2-tetrazoliumbromide (MTT) assay. For the cell counting assay, cells were seeded in a 24-well plate at a density of $2 \times 10^{4}$ cells/well in DMEM supplemented with $10 \%$ FBS. VSMCs were then infected with a different adenovirus at an MOI of 20-200 for $72 \mathrm{~h}$, or with various infection incubation times (1-6 days), and then synchronized in DMEM containing $0.2 \%$ FBS for $24 \mathrm{~h}$ and followed by adding $10 \mathrm{ng} / \mathrm{ml}$ human recombinant platelet-derived growth factor (PDGF)-BB (Peprotech Inc., Rocky Hill, NJ, USA) for $24 \mathrm{~h}$. Following treatment, cells were obtained by trypsinization (Gibco Life Technologies), and cell numbers were counted using a hemacytometer (Thermo Fisher Scientific, Waltham, MA, USA) with an inverted microscope, assessing three counts for each well and a mean value was acquired. Triplicate wells were assessed for each treatment and experiments were performed three times.

An MTT (Sigma-Aldrich, St. Louis, MO, USA) assay was performed as described previously (7). Briefly, VSMCs were subcultured into a 96 -well plate at $2 \times 10^{3}$ cells/well in DMEM alone and treated with as the above description in the cell counting assay. The absorbance value was measured at $570 \mathrm{~nm}$ using an enzyme-linked immnunosorbent assay (ELISA) reader (ELX800; BioTek Instruments, Inc., Winooski, VT, USA). Experiments were performed in triplicate $(n=3)$.

Statistical analysis. Data are presented as the mean \pm standard deviation. Differences were analyzed using SPSS version 15.0 software (SPSS Inc., Chicago, IL, USA). All values were analyzed using a one-way analysis of variance and the Q test. $\mathrm{P}<0.05$ was considered to indicate a statistically significant difference.

\section{Results}

Cloning and verifification of recombinant plasmid pDC316-hTK1-hTIMP1. The mCMV-hTIMP1 PCR fragment from pDC316-hTIMP1-EGFP was cloned to predigested plasmid pDC316-hTK1 by double-digestion (BglII/SalI), and subjected to ligation. The positive recombinant plasmid was extracted and a PCR reaction was performed using the aforementioned primers. The 1,338 bp fragment was observed on $1 \%$ agarose using a UV transilluminator (Fig. 1A). Subsequently, the recombinant plasmid was analyzed by double-digestion (BglII/SalI, Fig. 1B) and sequencing (Fig. 1C) providing the expected results. The hTK1 and hTIMP1 cDNA sequences are shown in Fig. 1C. A comparison of the hTK1 and hTIMP1 gene 


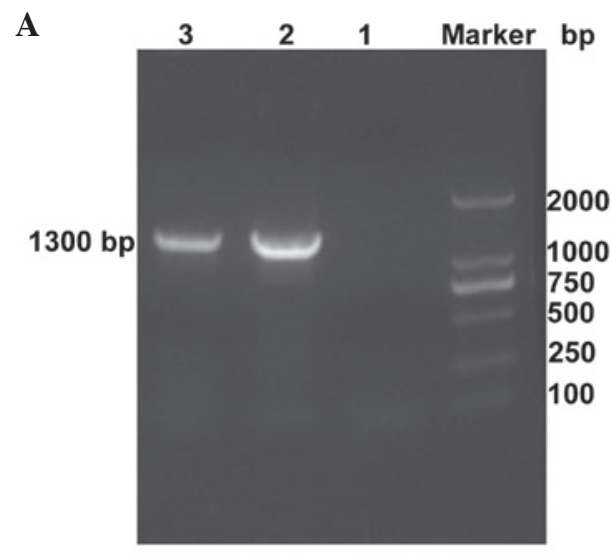

$\mathbf{B}$

C File: PDC316-httk-CMV-TimP(CMV-Profor)(20111223-R02-D06).ab1 $\begin{array}{ll}\text { Run Started: Dec 24, 2011, 0:58:25 } & \text { Signal G:1861 A:1518 T:1225 C:16 } \\ \text { Lane: } 42 \text { Base spacing 15.67 } & \text { 1284 bases in 16303 sans }\end{array}$

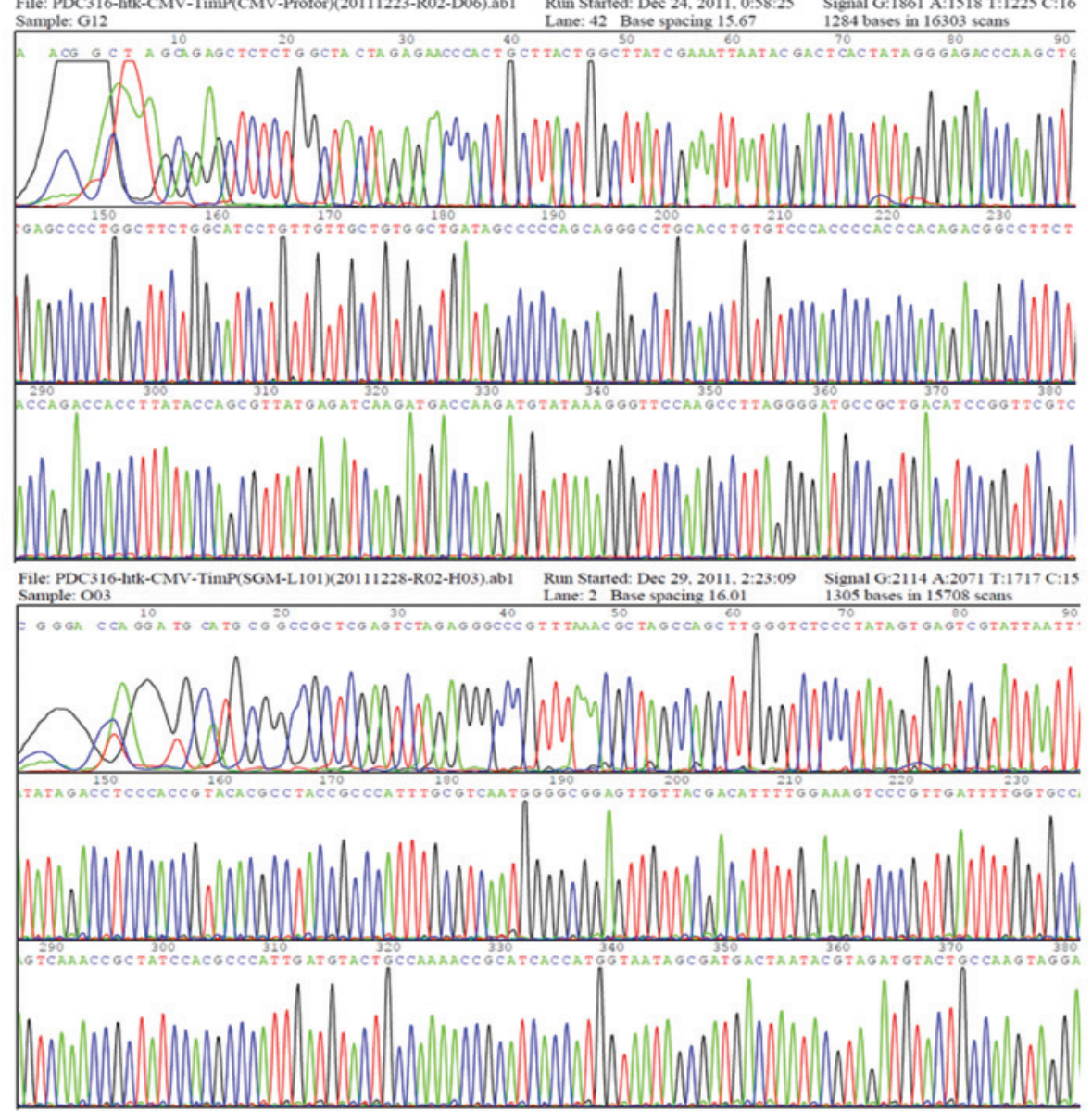

Figure 1. Identification of plasmid pDC316-hTK1-hTIMP1. (A) PCR reaction detection assay. Lane 1, negative control; Lane 2, mMCV-hTIMP1 (1,338 bp) gene obtained from pDC316-hTIMP1-EGFP plasmid (positive control); Lane 3, mMCV-hTIMP1 (1,338 bp) obtained from recombinant plasmid pDC316-hTK1-hTIMP1. (B) Double digestion assay by Bg1II/SalI: Lane 1, corresponding to double digested plasmid pDC316-hTK1-hTIMP1 (1,338 bp and $4.7 \mathrm{~kb}$ ). (C) Sequence analysis of the cloned PCR products confirmed hTK1 and hTIMP1 cDNA with primer pDC316-cexu-F. PCR, polymerase chain reaction; TK1, tissue kallikrein 1; TIMP1, tissue inhibitor of matrix metalloproteinase 1.

sequence with available sequences in gene bank revealed that the obtained sequence had a $100 \%$ homology with hTK1 and hTIMP1, and indicated that mMCV-hTIMP1 was correctly cloned into the plasmid pDC316-hTK1.

Packaging of recombinant adenovirus. With the aid of the AdMax system, the recombinant adenovirus Ad-hTK1-hTIMP1 was prepared in HEK293A cells. A viral plaque became visible at 9-11 days after transfection, as is shown in Fig. 2A. The target

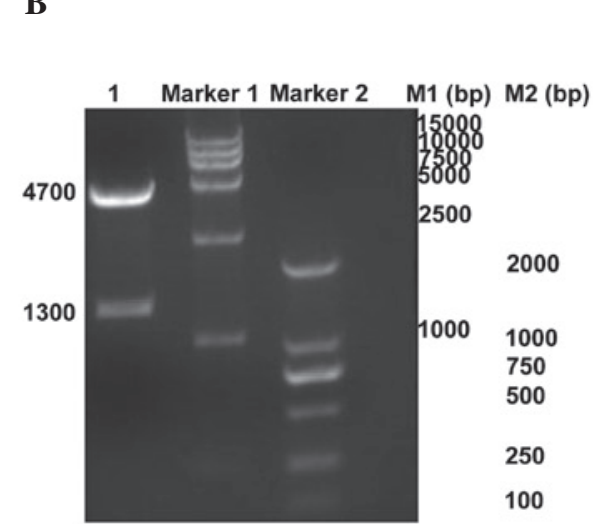


A

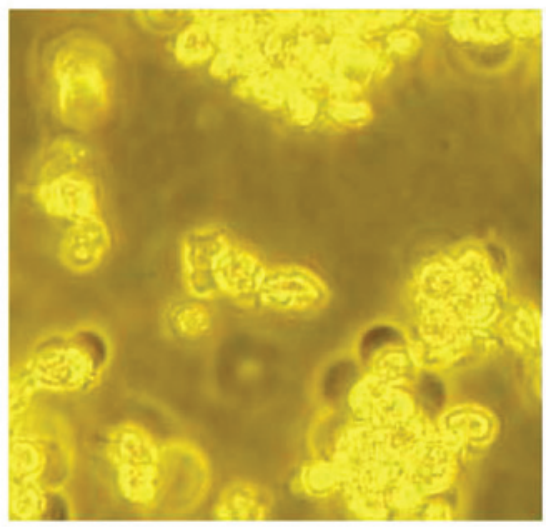

B

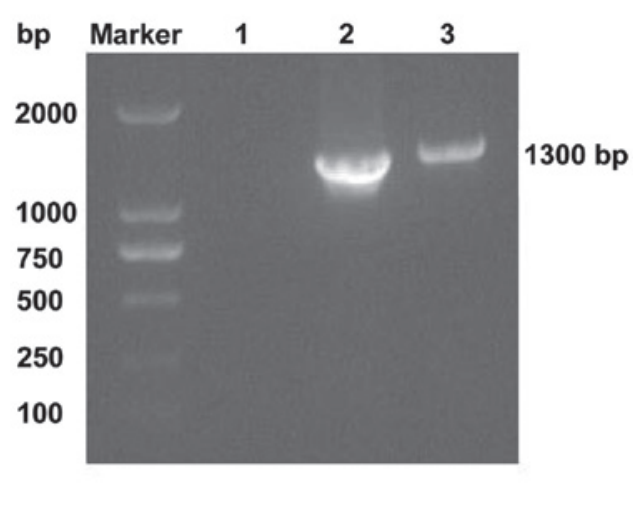

Figure 2. Packaging and verification of recombinant adenovirus Ad-hTK1-hTIMP1. (A) Morphological changes in Ad-hTK1-hTIMP1 transfected HEK293A cells (magnification, x100): The partial plaque formation of HEK293A cells after 10 days under an inverted microscope. (B) Identification of mMCV-hTIMP1 fragment using PCR. Lane 1, negative control; Lane 2: positive control, PCR product of pDC316- hTK1- hTIMP1; Lane 3: 1,338 bp PCR products detected from Ad-hTK1-hTIMP1. PCR, polymerase chain reaction; TK1, tissue kallikrein 1; TIMP1, tissue inhibitor of matrix metalloproteinase 1.

Ad-hTK1-hTIMP1 at an MOI of 20, 50, 100, 150 and 200, respectively, and mRNA expression was estimated by indirect RT-qPCR. As is shown in Fig. 3A, MOI-dependent expression was observed at 50-150 MOI at $72 \mathrm{~h}(\mathrm{P}<0.05$ 50-100 MOI, $\mathrm{P}<0.01150 \mathrm{MOI})$, respectively, whereas there was a lower level of expression in adenovirus-infected cells at 20-50 MOI $(\mathrm{P}>0.05)$. However, no significant difference was identified between $150 \mathrm{MOI}$ and $200 \mathrm{MOI}(\mathrm{P}>0.05)$.

The protein expression of Ad-hTK1-hTIMP1 was assessed using western blot analysis. As expected, the protein expression of hTK1 (33 kDa) and hTIMP1 (28.5 kDa) were detected in VSMCs. Ad-hTK1-hTIMP1 at an MOI of 20, 50, 100 and 200, as is shown in Fig. 3B and C, MOI-dependent expression was observed at 20-200 MOI at $72 \mathrm{~h}$ after infection $(\mathrm{P}<0.0550$ MOI, $\mathrm{P}<0.01$ 100-200 MOI), whereas there was a lower level of expression of hTK1 and hTIMP1 in the adenovirus-infected cells at $20 \mathrm{MOI}(\mathrm{P}>0.05)$. However, the mRNA and protein expression of hTK1 were markedly higher than that of hTIMP1 between 50 and $200 \mathrm{MOI}$ in the co-expression vector $(\mathrm{P}<0.05$ 50 MOI, $\mathrm{P}<0.01$ 100-200 MOI).

Therefore, 100-150 MOI of Ad-hTK1-hTIMP1 was accepted as an ideal level for subsequent experiments based on the evaluation of the transfection and expression efficiency assay.

Effects on cell growth and proliferation of Ad-hTK1-hTIMP1. It was investigated whether the co-expression vector of hTK1 and hTIMP1 genes had an effect on VSMC growth and proliferation.Primary VSMCs were infected with Ad-hTK1-hTIMP1 or Ad-EGFP, or left untreated as a control, and treated with or without PDGF-BB. The cell growth was measured using a cell counting assay. The number of cells induced by PDGF-BB was significantly increased compared with the control. However, Ad-hTK1-hTIMP1 significantly decreased cell numbers as compared with Ad-EGFP ( $\mathrm{P}<0.01)$. As is shown in Fig. 4A, Ad-hTK1-hTIMP1 had minor inhibitory effects on VSMC proliferation at lower concentrations (<20 MOI). Cell numbers were significantly reduced at concentrations $>50$ MOI of Ad-hTK1-hTIMP1 $(\mathrm{P}<0.05)$ as compared with $20 \mathrm{MOI}$. Compared with $100 \mathrm{MOI}$, the inhibition rate was $45.27 \%$ when
VSMCs were treated with 150 MOI $(\mathrm{P}<0.05)$. Cell number of cells infected with $200 \mathrm{MOI}$ of Ad-hTK1-hTIMP1 were no further decreased than those infected with $150 \mathrm{MOI}(\mathrm{P}>0.05)$. However, certain cells infected with Ad-hTK1-hTIMP1 at 200 MOI appeared to shrink and float, suggesting apoptosis and necrosis. Therefore, $150 \mathrm{MOI}$ of Ad-hTK1-hTIMP1 was the optimal dose for the present study.

As is shown in Fig. 4B, 150 MOI of Ad-hTK1-hTIMP1 had minor effects on VSMC proliferation following a short incubation time ( $<2$ days). By contrast, the cell numbers were significantly reduced after $>3$ days incubation in Ad-hTK1-hTIMP1-infected cells compared with 2 days $(\mathrm{P}<0.05)$. The peak inhibition rate was $46.6 \%$ when VSMCs were infected for 5 days. No significant difference in the inhibitory rate between the 6th and 7th day, as well as the 5 th day was observed $(\mathrm{P}>0.05)$. Certain cells infected with Ad-hTK1-hTIMP1 for 6 and 7 days appeared to shrink and float, suggesting apoptosis or necrosis. Therefore, it was found that 3 days after transfection was the optimal time for observation. These experiments suggested that within a given MOI (50-150 MOI) and time (2-5 days), the hTK1 and hTIMP1 co-expression inhibited PDGF-BB-stimulated VSMC growth in an MOI-dependent and time-dependent manner.

The synergistic inhibitory effects of the co-expression vector was subsequenlty investigated on cell proliferation. VSMCs were infected with Ad-hTK1-hTIMP1, Ad-hTK1, Ad-hTIMP1, or Ad-EGFP, and PDGF-BB-mediated cell growth was measured by cell count and MTT assay. PDGF induced significant increases in cell numbers and optical density (OD)values in PDGF-BB-stimulated and Ad-EGFP-infected VSMCs. As is shown in Fig. $4 \mathrm{C}$ and D, it was detected that the three recombinant adenoviruses significantly decreased cell number and OD values as compared with cells treated with PDGF-BB and Ad-EGFP $(\mathrm{P}<0.01)$. The peak inhibitory rates (analyzed by the OD value) of Ad-hTK1, Ad-hTIMP1 and Ad-hTK1-hTIMP1 were 27.4, 23.3 and 45.7\%, respectively. The inhibitory effect of Ad-hTK1-hTIMP1 on cell growth and proliferation exhibited a greater suppression than that of Ad-hTK1 and Ad-hTIMP1 (0.675 \pm 0.003 vs. 0.912 \pm 0.008 , $\mathrm{P}<0.05 ; 0.675 \pm 0.003$ vs. $0.951 \pm 0.005, \mathrm{P}<0.05)$. This result 
A

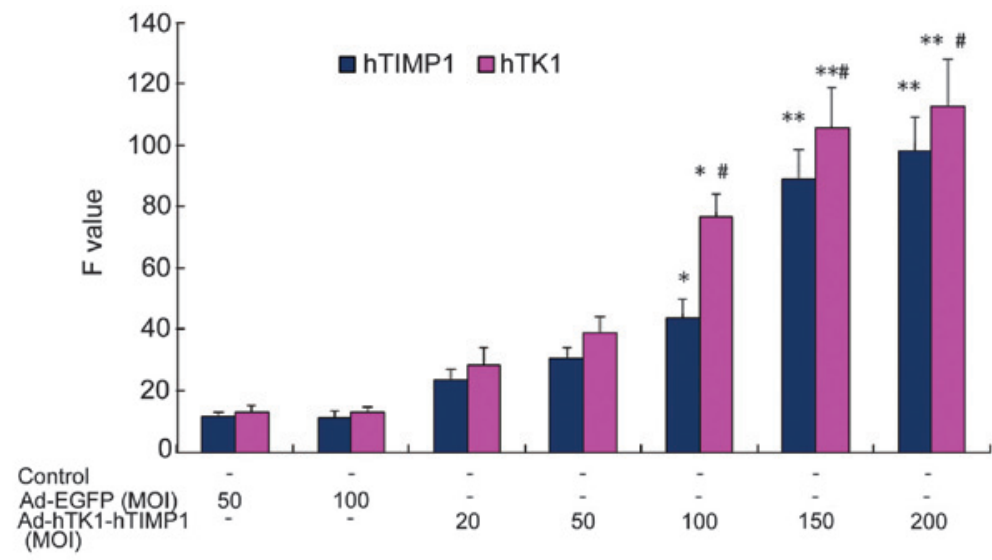

B

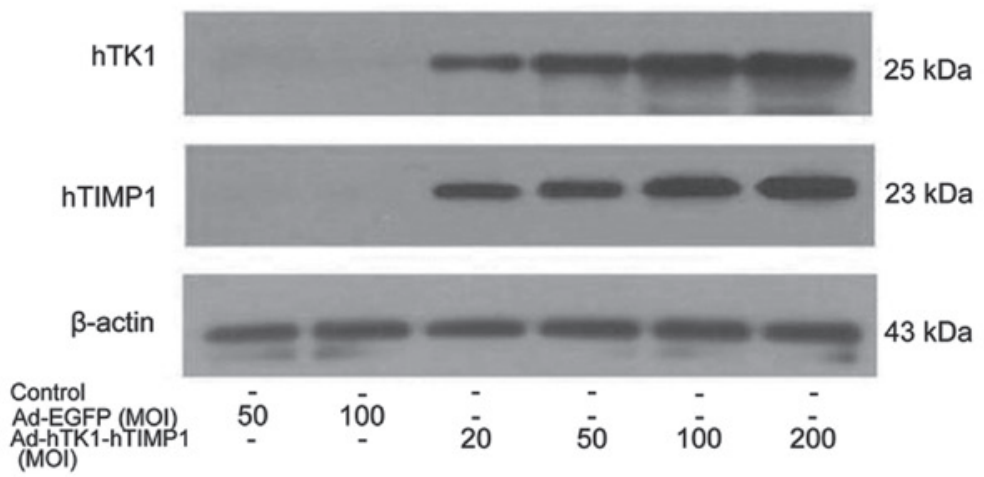

C

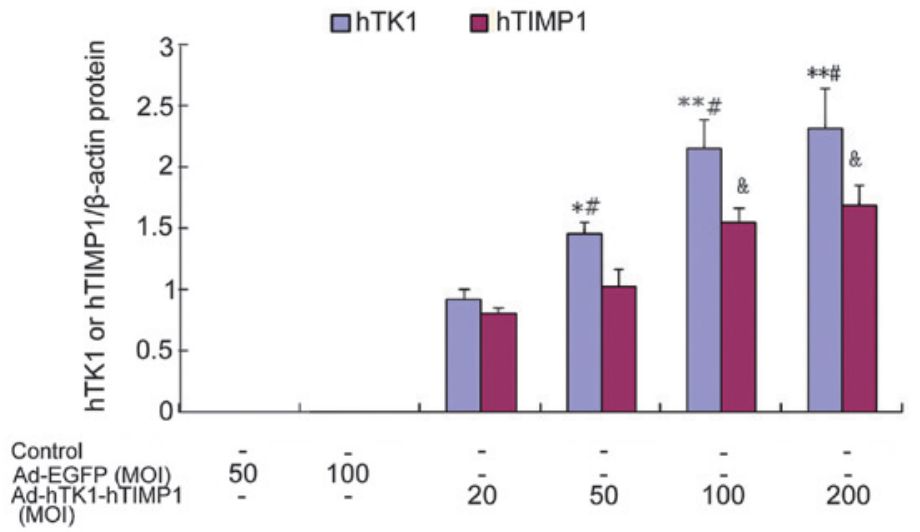

Figure 3. MOI-dependent co-expression of vascular smooth muscle cells infected with Ad-hTK1-hTIMP1. (A) Results of mRNA expression levels. Reseults are presented as the mean \pm standard deviation of 3 independent experiments ${ }^{*} \mathrm{P}<0.05$ and ${ }^{* *} \mathrm{P}<0.01$, compared with cells infected with 50 MOI. ${ }^{~} \mathrm{P}<0.01$, compared with mRNA expression of hTIMP1 in cells transferred with 100-150 MOI. (B and C) Expression of hTK protein assessed using western blot analysis ${ }^{*} \mathrm{P}<0.05$ and ${ }^{* *} \mathrm{P}<0.01$, compared with 20 MOI. ${ }^{\text {\& }} \mathrm{P}<0.01$, compared with $50 \mathrm{MOI} .{ }^{*} \mathrm{P}<0.01$, compared with expression of hTIMP1 protein in cells infected with 50-200 MOI. TK1, tissue kallikrein 1; TIMP1, tissue inhibitor of matrix metalloproteinase 1.

suggests a possible association of the synergistic suppression of proliferation by co-expression of the hTK1 and hTIMP1 genes. No significant differences were observed between cells treated with Ad-hTK1 and Ad-hTIMP1, and Ad-EGFP and PDGF-BB only.

\section{Discussion}

Vascular remodeling occurs following the abnormal migration and proliferation of VSMCs, as well as the ECM rearrangement (22). Numerous risk factors are involved in the development of the pathological process. Multigene-based combination therapy represents an effective practice in atherosclerosis, which may achieve greater therapeutic benefits (23). Recent studies have reported that TK1 is important in reducing blood pressure, improving cardiac remodeling, renal injury and ischemic stroke, and preventing the long-term in-stent restenosis (1-8). Subsequent studies have demonstrated that TIMP1, as a regulator of MMPs, exhibits a number of beneficial effects on the inhibition of vascular remodeling by blocking the activation of MMPs, supressing VSMC migration and destabilizing vessel differentiation $(14,24)$. However, the therapeutic effect of the combination treatment of TK1 and TIMP1 on VSMC proliferation remains to be investigated. Based on the anti-restenosis properties of TK1 and TIMP1, it was hypothesized that the combination of TK1 and TIMP1 
A

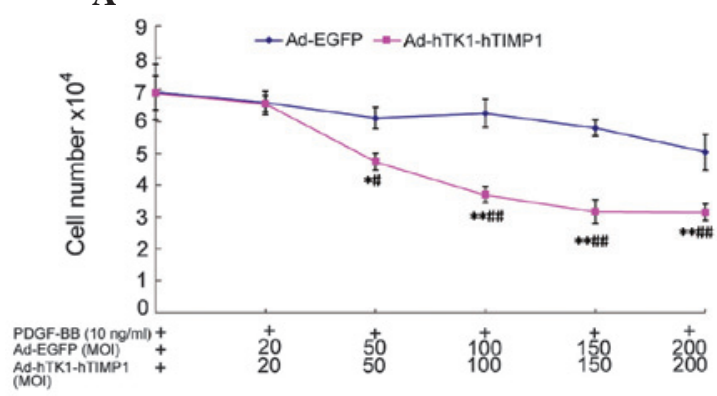

C

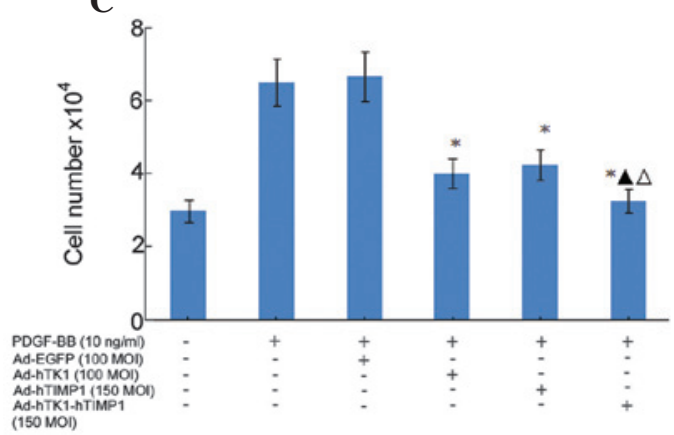

B

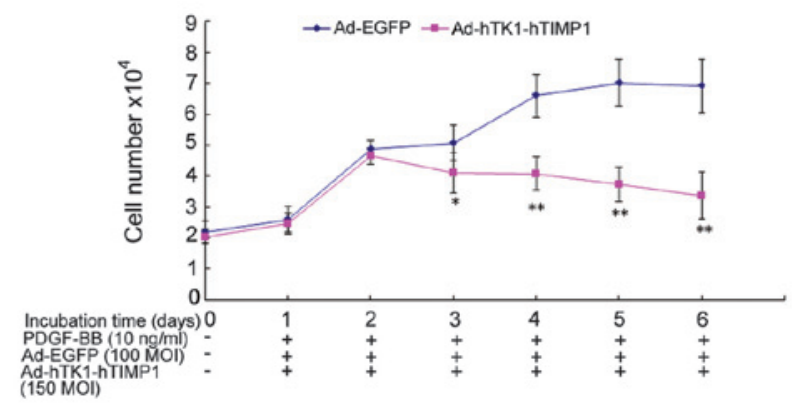

D

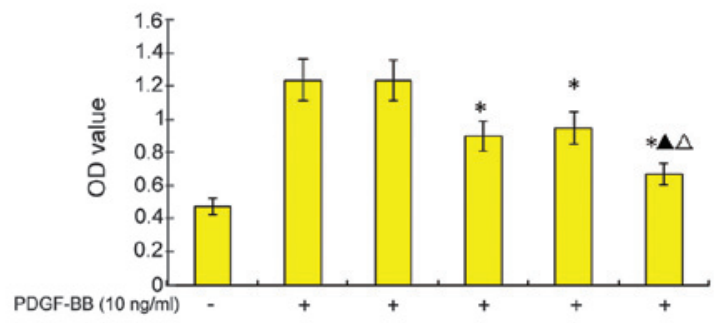

Figure 4. Inhibitory effects of Ad-hTK1-hTIMP1 on cell growth and proliferation in VSMCs. (A) Concentration-dependent inhibitory effects of co-expression vector on VSMC growth assessed by cell counting. Cells treated with $0.2 \%$ fetal bovine serum were used as a control. ${ }^{*} \mathrm{P}<0.05$ and ${ }^{* *} \mathrm{P}<0.01$, compared with cells infected with 20 MOI Ad-hTK1-hTIMP1; ${ }^{\#} \mathrm{P}<0.05$ and ${ }^{\# \#} \mathrm{P}<0.05$, as compared with the cells infected with Ad-EGFP. (B) Time-dependent (1-6 days) inhibitory effects of co-expression vector on VSMC growth assessed by cell counting. ${ }^{*} \mathrm{P}<0.05,{ }^{* *} \mathrm{P}<0.01$, compared with Ad- hTK1-hTIMP1 for 2 days. (C and D) Inhibitory proliferation effects of co-expression vector evaluated using cell counting and a 3-[4,5-dimethy1-2-thiazolyl]-2,5-dipheny1-2-tetrazoliumbromide assay. ${ }^{*} \mathrm{P}<0.01$, compared with cells stimulated with platelet-derived growth factor-BB only; ${ }^{\mathbf{\Delta}} \mathrm{P}<0.05$ as compared with cells infected with Ad-hTK1; ${ }^{\Delta} \mathrm{P}<0.05$ as compared with cells infected with Ad-hTIMP1. TK1, tissue kallikrein 1; TIMP1, tissue inhibitor of matrix metalloproteinase 1; VSMCs, vascular smooth muscle cells.

would exert an enhanced or synergistic effect on inhibiting VSMC proliferation.

Adenoviral vectors harboring therapeutic genes have been used successfully for gene transfer in vitro and in vivo $(16,17)$. In the present study, the recombinant plasmid, pDC316-hTK1-hTIMP1 was successfully constructed and verified using PCR, double digestion and sequencing assays. The recombinant adenovirus, Ad-hTK1-hTIMP1 was successfully packaged into HEK293A cells with the aid of a highly efficient Cre/loxP-based system $(7,18,25)$. The co-expression vector contains hTK1 and hTIMP1 double stranded cDNA by means of a double mCMV promoter, and avoids the production of a fusion protein. The titer of the co-expression vector was $1.26 \times 10^{10} \mathrm{IU} / \mathrm{ml}$ as assessed using the TCID50 method. Furthermore, it was demonstrated that there was an increase in hTK1 and hTIMP1 transcription and protein when VSMCs were infected with the co-expression vector, which occurred in a dose (50-150 MOI) and time-dependent (2-5 day) manner. No significant differences were identified in the expression levels of hTK1 and hTIMP1 in a 150 MOI co-expression vector compared with a 200 MOI co-expression vector. However, the expression level of hTK1 was greater compared with that of hTIMP1 in the co-expression vector transfected with VSMCs. The present study confirmed that the co-expression vector carrying full length hTK1 and hTIMP1 cDNA could successfully co-express hTK1 and hTIMP1 protein in VSMCs.

Our previous study also demonstrated that the over-expression of the hTK1 and hTIMP1 genes mediated by Ad-hTK1 and Ad-hTIMP1 could inhibit the proliferation of VSMCs induced by PDGF-BB, where the peak inhibitory rate was 30.2 and $23.2 \%$, respectively (7). TIMP1 has been observed to mediate the inhibitory effect of interleukin (IL)- 6 on the proliferation of a hepatocellular carcinoma cell line (26). To further examine the synergistic effects of these genes and whether the co-expression vector inhibits the proliferation of VSMCs, PDGF-BB-stimulated cells were treated with Ad-hTK1-hTIMP1, Ad-hTK1 and Ad-hTIMP1, and were assessed using cell counting and an MTT assay. It was demonstrated that the co-expression vector supressed cell growth and proliferation in an MOI-dependent (50-150 MOI) and time-dependent manner (2-5 days), with a peak inhibitory rate of $48.2 \%$ (150 MOI) and 39.7\% (5 days). It was also demonstrated that the co-expression vector synergistically inhibited VSMC growth and proliferation. Zhao et al (27) also demonstrated that the adenovirus-mediated inhibitor of growth family, member 4 and IL-24 double tumor suppressor gene co-expression enhances the antitumoral activity in human breast cancer cells, reduces side effects from vectors, and has satisfactory prospects for clinical application. The study of Deng et al (28) implied that a molecular therapy combining two or more functionally synergistic tumor suppressors may constitute a novel and effective strategy for cancer treatment. However, how protected effective function contributed to improve vascular remodeling by means of a co-expression vector remains to be further examined and elucidated.

In conclusion, the present data demonstrated that hTK1 and hTIMP1 full-length cDNA in a single adenovirus vector may be successfully constructed and achieve co-expression of 
hTK1 and hTIMP1 proteins, independently and abundantly. There was a synergistic inhibitory proliferative effect on VSMCs infected with the co-expression vector, suggesting that it may be an effective therapeutic strategy for vascular remodeling disease.

\section{Acknowledgements}

The present study was supported by grants from the Natural Science Foundation of China (grant no. 81373785), the Natural Science Foundation of Fujian Province (grant nos. 2011J01134 and 2010J01127) and the Projection of Excellent Yang Doctor training plan in Fujian Provincial Health System (grant no. 2013-ZQN-ZD-2).

\section{References}

1. Ashley PL and MacDonald RJ: Tissue-specific expression of kallikrein-related genes in the rat. Biochemistry 24: 4520-4527, 1985.

2. Gerald WL, Chao J and Chao L: Sex dimorphism and hormonal regulation of rat tissue kallikrein mRNA. Biochim Biophys Acta 867: 16-23, 1986.

3. Yao Y, Sheng Z, Li Y, Yan F, Fu C, Li Y, Ma G, Liu N, Chao J and Chao L: Tissue kallikrein promotes cardiac neovascularization by enhancing endothelial progenitor cell functional capacity. Hum Gene Ther 23: 859-870, 2012.

4. Yin $\mathrm{H}$, Chao L and Chao J: Nitric oxide mediates cardiac protection of tissue kallikrein by reducing inflammation and ventricular remodeling after myocardial ishchemia/reperfusion. Life Sci 82: 156-165, 2008.

5. Liu Y, Bledsoe G, Hagiwara M, Yang ZR, Shen B, Chao L and Chao J: Blockade of endogenous tissue kallikrein aggravates renal injury by enhancing oxidative stress and inhibiting matrix degradation. Am J Physiol Renal Physiol 298: F1033-F1040, 2010.

6. Lan W, Yang F, Liu L, Yin Q, Li M, Li Z, Sang H, Xu G, Ma M, Zhang Z, et al: Tissue kallikrein preventing the restenosis after stenting of symptomatic MCA atherosclerotic stenosis (KPRASS). Int J Stroke 9: 533-535, 2014.

7. Yu HZ, Xie LD, Zhu PL, Xu CS and Wang HJ: Human tissue kallikrein 1 gene delivery inhibits PDGF-BB-induced vascular smooth muscle cells proliferationand upregulates the expressions of p27Kip1 and p2lCip1. Mol Cell Biochem 360: 363-371, 2012.

8. Yu HZ, Xie LD, Zhu PL, Xu CS, Wang HJ and Li TY: Effects of human tissue kallikrein 1 gene delivery on carotid artery neointima formation after balloon angioplasty in spontaneously hypertensive rats. Zhonghua Xin Xue Guan Bing Za Zhi 38: 67-71, 2010 (In Chinese).

9. Heagerty AM, Heerkens EH and Izzard AS: Small artery structure and function in hypertension. J Cell Mol Med 14: $1037-1043,2010$

10. Intengan HD and Schiffrin EL: Vascular remodeling in hypertension: Roles of apoptosis, inflammation and fibrosis. Hypertension 38: 581-587, 2001.

11. Zitka O, Kukacka J, Krizkova S, Huska D, Adam V, Masarik M, Prusa R and Kizek R: Matrix metalloproteinases. Curr Med Chem 17: 3751-3768, 2010.

12. Papazafiropoulou A and Tentolouris N: Matrix metalloproteinases and cardiovascular diseases. Hippokratia 13: 76-82, 2009.
13. Docherty AJ, Lyons A, Smith BJ, Wright EM, Stephens PE, Harris TJ, Murphy G and Reynolds JJ: Sequence of human tissue inhibitor of metalloproteinases and its identity to erythroid-potentiating activity. Nature 318: 66-69, 1985.

14. Dollery CM, Humphries ES, McClelland A, Latchman DS and McEwan JR: Expression of tissue inhibitor of matrix metalloproteinases 1 by use of an adenoviral vector inhibits smooth muscle cell migration and reduces neointimal hyperplasia in the rat model of vascular balloon injury. Circulation 99: 3199-3205, 1999.

15. Pei Z, Chu L, Zou W, Zhang Z, Qiu S, Qi R, Gu J, Qian C and Liu X: An oncolytic adenoviral vector of Smac increases antitumor activity of TRAIL against HCC in human cells and in mice. Hepatology 39: 1371-1381, 2004.

16. Shen Y, Muramatsu SI, Ikeguchi K, Fujimoto KI, Fan DS, Ogawa M, Mizukami H, Urabe M, Kume A, Nagatsu I, et al: Triple transduction with adeno-associated virus vectors expressing tyrosine hydroxylase, aromatic-L-amino-acid decarboxylase and GTP cyclohydrolase I for gene therapy of Parkinson's disease. Hum Gene Ther 11: 1509-1519, 2000.

17. Ngoi SM, Chien AC and Lee CG: Exploiting internal ribosome entry sites in gene therapy vector design. Curr Gene Ther 4: 15-31, 2004.

18. Denes L, Entz L and Jancsik V: Restenosis and therapy. Int J Vasc Med 406236, 2012.

19. Ng P, Parks RJ, Cummings DT, Evelegh CM, Sankar U and Graham FL: A high-efficiency Cre/loxP-based system for construction of adenoviral vectors. Hum Gene Ther 10: 2667-2672, 1999

20. Maeda M, Namikawa K, Kobayashi I, Ohba N, Takahara Y, Kadono C, Tanaka A and Kiyama H: Targeted gene therapy toward astrocytoma using a Cre/loxP-based adenovirus system. Brain Res 1081: 34-43, 2006

21. Chen HF, Xie LD and Xu CS: The signal transduction pathways of heat shock protein 27 phosphorylation in vascular smooth muscle cells. Mol Cell Biochem 333: 49-56, 2010.

22. Gariepy J, Massonneau M, Levenson J, Heudes D and Simon A: Evidence for in vivo carotid and femoral wall thickening in human hypertension. Groupe de Prévention Cardio-vasculaire en Médecine du Travail. Hypertension 22: 111-118, 1993.

23. Wilson DR: Viral-mediated gene transfer for cancer treatment. Curr Pharm Biotechnol 3: 151-164, 2002.

24. Eefting D, Seghers L, Grimbergen JM, de Vries MR, de Boer HC, Lardenoye JW, Jukema JW, van Bockel JH and Quax PH: A novel urokinase receptor-targeted inhibitor for plasmin and matrix metalloproteinases suppresses vein graft disease. Cardiovasc Res 88: 367-375, 2010.

25. Jiang M, Shi W, Zhang Q, Wang X, Guo M, Cui Z, Su C, Yang Q, Li Y, Sham J, et al: Gene therapy using adenovirus-mediated full-length anti-HER-2 antibody for HER-2 overexpression cancers. Clin Cancer Res 12: 6179-6185, 2006.

26. Guo SY, Shen X, Yang J, Yuan J, Yang RL, Mao K, Zhao DH and Li CJ: TIMP-1 mediates the inhibitory effect of interleukin- 6 on the proliferation of a hepatocarcinoma cell line in a STAT3-dependent manner. Braz J Med Biol Res 40: 621-631, 2007.

27. Zhao YD, Li ZY, Sheng WH, Miao J and Yang J: Adenovirus-mediated ING4/IL-24 double tumor suppressor gene co-transfer enhances antitumor activity in human breast cancer cells. Oncol Rep 28: 1315-1324, 2012.

28. Deng WG, Kawashima H, Wu G, Jayachandran G, Xu K, Minna JD, Roth JA and Ji L: Synergistic tumor suppression by coexpression of FUS1 and p53 is associated with down-regulation of murine double minute- 2 and activation of the apoptotic protease-activating factor 1-dependent apoptotic pathway in human non-small cell lung cancer cells. Cancer Res 67: 709-717, 2007. 